\title{
The Effect of Imatinib Treatment Duration on the Quality of the Life of Patients with Chronic Myeloid Leukemia
}

\author{
Katerina Skrivanova ${ }^{1 *}$, Marcela Bendova', Ladislav Dusek ${ }^{2}$, Daniela Zackova ${ }^{3}$, Zdenek Racil ${ }^{3}$ and Jiri Mayer ${ }^{3,4}$ \\ ${ }^{1}$ Department of Psychology and Psychosomatics, Faculty of Medicine, Kamenice 3, 625 00, Masaryk University, Brno, Czech Republic \\ ${ }^{2}$ Institute of Biostatistics and Analyses Masaryk University, Brno, Czech Republic \\ ${ }^{3}$ Department of Internal Medicine-Hematology and Oncology, Masaryk University and University Hospital Brno, Brno, Czech Republic \\ ${ }^{4}$ Central European Institute of Technology (CEITEC), Brno, Czech Republic
}

\begin{abstract}
Purpose: The objective of this study was to evaluate the quality of life (QL) as well as the frequency and severity of depression in patients with chronic phase chronic myeloid leukemia (CP-CML) in relation to the duration of Imatinib treatment.
\end{abstract}

Methods: A detailed analysis of 56 adult patients with $\mathrm{CP} C M L$ divided into groups according to the length of Imatinib treatment was performed as follows: Group A ( $n=28)$ - patients treated for 15 months (median); and Group B ( $n=28$ )-patients treated for 50 months (median). All used questionnaires EORTC QLQ-C30 (version 2), SF 36 and BDI were localized and validated.

Results: The results from the QL questionnaires found a trend in the improvement in the QL of patients treated with Imatinib for 50 months (median) compared to patients treated for 15 months (median) only. However, a statistically significant improvement in the QL of patients with longer Imatinib treatment was found only in patients without co-existing invalidity and/or rheumatic disease. Moreover, these two comorbidities increased the level of depressive symptoms in patients with CML during the early period of Imatinib therapy.

Conclusion: We found a trend in the improvement in the QL of patients with CML in relation to the duration of Imatinib therapy. Statistically significant improvement in the QL of patients with longer Imatinib treatment was found in patients without co-existing invalidity and/or rheumatic disease only.

Keywords: Quality of life; CML; Comorbidities; Imatinib; Cancer treatment; Oncology

Abbreviations: QL: Quality of Life; CP: Chronic Phase; CML: Chronic Myeloid Leukemia; EORTC QLQ-C30 European Organization for Research and Treatment of Cancer Quality of Life Questionnaire; SF-36: Short Form-36; BDI: Beck Depression Inventory

\section{Introduction}

Improvements in the outcomes of cancer treatment and the real possibility of long-term survival have increased the attention placed on the quality of life (QL) and on its monitoring in patients with malignancies. The QL definition is derived from a concept that describes four areas of a full-value life for an individual-physical, psychological, social status, and spiritual aspects [1]. The QL in cancer patients is influenced not only by the consequences of the disease itself, but also by side effects of the treatment [2]. Moreover, Houts et al. highlighted the negative impact of depression on QL in oncological patients and the importance of its surveillance [3].

Chronic myeloid leukemia (CML) is a myeloproliferative disease that represents $15 \%-25 \%$ of all leukemias. The disease arises as a result of a reciprocal translocation between chromosome 22 and chromosome 9, the so-called Philadelphia translocation [4]. This rearrangement joins the c-ABL1 gene on chromosome 9 and BCR on chromosome 22 creating a BCR-ABL1 fusion gene, which codes for $190-230 \mathrm{kDa}$, $\mathrm{Bcr}-\mathrm{Abl}$ fusion protein with elevated tyrosine kinase activity. The upregulated Bcr-Abl fusion protein includes the tyrosine kinase domain, which likely contributes to dysregulation of the mechanism of cellular signals transduction, normally involved in controlling apoptosis, proliferations, and cell-cell adhesions, and thus promoting leukemogenesis [5]. The abrogation of Bcr-Abl function has become a model for development of targeted therapies. The enzymatic inhibition of Bcr-Abl using Imatinib was showed to possess potent in vitro and in vivo activities in preclinical studies and its long-term efficacy and safety was confirmed in clinical trials [6-8].

Imatinib has changed the paradigm of treating CML, and today it is a standard of care as an initial therapy for patients with this malignancy, providing a significant probability for long-term survival [9]. Imatinib response evaluation and monitoring according to valid international recommendations is a critical point of this successful therapy $[10,11]$.

There are only limited data analyzing QL in patients with CML treated with IMA, with the exception of data presented by Hahn et al., which analyzed patients from the IRIS study [12]. However, there are no data available to date that have analyzed the influence of the length of Imatinib treatment on the QL of patients with CML. Thus, the objective of this study was to evaluate the QL as well as the frequency and level of depression in patients with CML in relation to the duration of Imatinib therapy.

*Corresponding author: Katerina Skrivanova, Department of Psychology and Psychosomatics, Faculty of Medicine, Kamenice 3, 625 00, Masaryk University, Brno, Czech Republic, Tel: 54949-28904821; E-mail: kskrivan@med.muni.cz

Received July 30, 2013; Accepted October 16, 2013; Published October 22, 2013

Citation: Skrivanova K, Bendova M, Dusek L, Zackova D, Racil Z, et al. (2013) The Effect of Imatinib Treatment Duration on the Quality of the Life of Patients with Chronic Myeloid Leukemia. J Blood Disorders Transf 4: 167. doi: 10.4172/2155-9864.1000167

Copyright: (C) 2013 Skrivanova K, et al. This is an open-access article distributed under the terms of the Creative Commons Attribution License, which permits unrestricted use, distribution, and reproduction in any medium, provided the original author and source are credited. 
Citation: Skrivanova K, Bendova M, Dusek L, Zackova D, Racil Z, et al. (2013) The Effect of Imatinib Treatment Duration on the Quality of the Life of Patients with Chronic Myeloid Leukemia. J Blood Disorders Transf 4: 167. doi: 10.4172/2155-9864.1000167

Page 2 of 5

\section{Methods}

This study was retrospective and lateral. We analyzed 56 patients with CP-CML who were treated at the Department of Internal Medicine-Hematology and Oncology, Masaryk University, and at the University Hospital Brno. All patients gave their informed consent with participation and the study was approved by the IRB. Two methods of self-evaluation scales were used to score QL: EORTC QLQ-C30 (version 2) and SF-36 [13-15]. Questionnaires EORTC-QLQ-C30 (version 2) and SF-36 were scored according to valid recommendations [16]. In accordance with the generally accepted recommendations, it is possible to consider a difference of at least 10 percentage points in the QL score as clinically relevant [16].

For the evaluation of comorbidities and their importance, a retrospective analysis of patients' documentation was performed by hematologists. These other potential co-factors of quality of life were monitored at the time of the diagnosis, during Imatinib therapy, and at the time of testing. The presence of comorbidity was scored and tested as binary variables (YES/NO), and its importance was scaled as 0,1 , and 2 (without specific comorbidity, the presence of a mild degree of specific comorbidity, and the presence of a severe degree of specific comorbidity, respectively).

The influence of the length of Imatinib therapy on QL was analyzed in the total sampling as well as in groups that were divided according to significant factors influencing the QL score. ANCOVA analysis was used for detection of an effect of co-factors (such as age, BMI, sex, and the presence of comorbidities, including rheumatic diseases and

\begin{tabular}{|c|c|c|}
\hline Parameter & $\begin{array}{l}\text { Group A: patients with Imatinib therapy } \\
\text { for } 15 \text { months }(\mathrm{N}=28)\end{array}$ & $\begin{array}{l}\text { Group B: patients with Imatinib therapy for } 50 \text { months } \\
\qquad(\mathrm{N}=28)\end{array}$ \\
\hline Male sex & $\mathrm{N}=15(53.6 \%)$ & $\mathrm{N}=13(46.4 \%)$ \\
\hline Age $(y r s)^{1}$ & $60(33-80)$ & $61(33-74)$ \\
\hline Age $\geq 60$ yrs & $\mathrm{N}=16(57.1 \%)$ & $\mathrm{N}=16(57.1 \%)$ \\
\hline BMI $\left(\mathrm{kg} \cdot \mathrm{m}^{-2}\right)$ & $27.2(16.8-38.1)$ & $27.4(16.0-40.0)$ \\
\hline Other malignancies & $\mathrm{N}=1(3.6 \%)$ & $\mathrm{N}=2(7.1 \%)$ \\
\hline Other cancer therapy & $\mathrm{N}=1(3.6 \%)$ & $\mathrm{N}=0(0.0 \%)$ \\
\hline Cardiac problems & $\mathrm{N}=11(39.3 \%)$ & $\mathrm{N}=7(25.0 \%)$ \\
\hline Stroke & $\mathrm{N}=0(0.0 \%)$ & $\mathrm{N}=1(3.6 \%)$ \\
\hline Rheumatic disease & $\mathrm{N}=5(17.9 \%)$ & $\mathrm{N}=5(17.9 \%)$ \\
\hline Gastrointestinal disorders & $\mathrm{N}=7(25.0 \%)$ & $\mathrm{N}=8(28.6 \%)$ \\
\hline Invalidity & $\mathrm{N}=12(42.9 \%)$ & $\mathrm{N}=8(28.6 \%)$ \\
\hline Rheumatic disease and/or invalidity & $\mathrm{N}=17(60.7 \%)$ & $\mathrm{N}=16(57.1 \%)$ \\
\hline Prior CML treatment & $\mathrm{N}=10(35.7 \%)$ & $\mathrm{N}=19(67.9 \%)$ \\
\hline Interferon- $\alpha$ & $\mathrm{N}=6$ & $\mathrm{~N}=19$ \\
\hline Hydroxyurea & $N=10$ & $\mathrm{~N}=17$ \\
\hline Other treatment & $\mathrm{N}=5$ & $N=11$ \\
\hline $\begin{array}{l}\text { Imatinib treatment }(\mathrm{mg} / \text { day }) \\
-\quad \text { Starting dose, } \mathrm{mg} / \text { day }\end{array}$ & $400(200-400)$ & $400(300-400)$ \\
\hline Baseline dose (mg/day) & $400(250-800)$ & $400(100-800)$ \\
\hline \multicolumn{3}{|l|}{ Disease phase } \\
\hline Chronic & $\mathrm{N}=27(96.4 \%)$ & $\mathrm{N}=27(96.4 \%)$ \\
\hline Not applicable & $\mathrm{N}=1(3.6 \%)$ & $\mathrm{N}=1(3.6 \%)$ \\
\hline \multicolumn{3}{|l|}{ Sokal risk group } \\
\hline Low & $\mathrm{N}=7(25.0 \%)$ & $\mathrm{N}=9(32.1 \%)$ \\
\hline Intermediate & $\mathrm{N}=10(35.7 \%)$ & $\mathrm{N}=9(32.1 \%)$ \\
\hline High & $\mathrm{N}=6(21.4 \%)$ & $\mathrm{N}=3(10.7 \%)$ \\
\hline Not applicable & $\mathrm{N}=5(17.9 \%)$ & $\mathrm{N}=7(25.0 \%)$ \\
\hline \multicolumn{3}{|l|}{ Imatinib therapy status } \\
\hline success & $\mathrm{N}=21(75.0 \%)$ & $\mathrm{N}=25(89.3 \%)$ \\
\hline failure* & $\mathrm{N}=7(25.0 \%)$ & $\mathrm{N}=3(10.7 \%)$ \\
\hline \multicolumn{3}{|l|}{${ }^{*}$ Reason of failure } \\
\hline Intolerance & $N=1$ & $N=1$ \\
\hline Resistance & $N=4$ & $\mathrm{~N}=1$ \\
\hline Mutation & $N=3$ & $\mathrm{~N}=0$ \\
\hline Progression & $\mathrm{N}=2$ & $\mathrm{~N}=1$ \\
\hline
\end{tabular}

1 Continuous variable are summarized as median supplied with min -max range (in parentheses).

Binary variables are expresses as $\mathrm{N}(\%)$ of a respective item.

*Imatinib failure was defined as a progression to advanced phase of the disease, loss of hematologic or cytogenetic response, lack of hematologic or cytogenetic response achievement at the defined time points, presence of Imatinib-resistant Bcr-Abl mutation, discontinuation of Imatinib treatment because of intolerance or death from any cause, whichever came first [11]

Table 1: Patient Demographic and Clinical Characteristics. 
Citation: Skrivanova K, Bendova M, Dusek L, Zackova D, Racil Z, et al. (2013) The Effect of Imatinib Treatment Duration on the Quality of the Life of Patients with Chronic Myeloid Leukemia. J Blood Disorders Transf 4: 167. doi: 10.4172/2155-9864.1000167

Page 3 of 5

invalidity) on patients' QL scores. A Pearson correlation was used to determine a potential effect of the presence of different comorbidities (gastrointestinal disorders, joint pain or muscle spasms) on patients' QL scores.

In addition, we used the structured clinical interview and Beck Depression Inventory (BDI) for the diagnosis of depression [17]. The clinical interview included seven areas, of which we were interested in permanent somatic problems, depression, knowledge about CML as a disease, social status, active employment, future prospects, ability to communicate about the disease, and cooperation with the community of other patients with CML treated with Imatinib for the analysis of structured interviews, we used a common approach based on the process of classification and description. All of the statistical analyses were performed with SPSS v.12.0.1 software [18]

\section{Results and Discussion}

The basic demographic and clinical characteristics of the patient groups are shown in Table 1. The patients were divided into two groups according to the length of Imatinib treatment. Group A included 28 patients ( $36 \%$ were pretreated prior to receiving Imatinib) who were treated with Imatinib for a median duration of 15 months (range 10-19 months), and Group B included 28 patients (68\% were pretreated prior to receiving Imatinib) who were treated with Imatinib for a median duration of 50 months (range 26-57 months).

An overview of the QL scores according to the Short Form Health Survey (SF-36) as well as European Organization for Research and Treatment of Cancer Quality Of Life Questionnaire (EORTC QLQ-C30) suggested that the QL in our patient groups ranged from very good up to substantially low QL. Moreover, our results showed a trend toward an improved QL in the group receiving long-term Imatinib treatment compared to the group receiving Imatinib for a shorter time. However, these overall differences were not statistically significant $(\mathrm{p}=0.284)$.

We verified other stratifying factors (other than the length of Imatinib therapy) as well, which may have a substantial impact on the QL score, including gastrointestinal disorders, rheumatic diseases, BMI, sex, and invalidity (defined as incapability due to a chronic illness or disability). Age-adjusted analysis of the QL scores showed that only rheumatic diseases and/or invalidity had a substantial impact on most of the QL parameters (Table 2). Patients with invalidity and/or rheumatic disease had significantly lower parameters of QL scores, such as an overall health condition $(\mathrm{p}=0.018)$, physical functions $(\mathrm{p}=0.012)$, and related symptoms $(\mathrm{p}=0.025)$.

Based on these findings (Table 2) and the observation of a better QL trend in the group receiving long-term Imatinib treatment, an age-adjusted analysis was performed in both groups of patients after stratification according to the presence of invalidity and/or rheumatic disease. This analysis showed a statistically significant improvement in QL in patients with longer Imatinib therapy; however, this was only observed in individuals without co-existing invalidity and/or rheumatic disease. Both questionnaires (SF-36 and QLQ-C30) provided the same results (Table 3 ).

Bayliss et al. previously analyzed the relationship between QL and comorbidities in persons with chronic medical conditions and found comorbidity as a significant factor that influenced QL [19]. Therefore, based on these previous findings, we analyzed the effects of other comorbidities (i.e. gastrointestinal disorders, joint pain and muscle cramps) as other potential factors that could lead to changes in QL. No significant correlation with the observed changes in patients' QL was observed for any of the mentioned factors. The differences in the occurrence of these factors between the groups of patients ranged

\begin{tabular}{|c|c|c|c|c|c|}
\hline $\begin{array}{l}\text { Parameter } \\
\text { (cofactor) }\end{array}$ & $\begin{array}{c}\text { SF-36: } \\
\text { physical health }\end{array}$ & $\begin{array}{c}\text { SF-36 } \\
\text { mental health }\end{array}$ & $\begin{array}{l}\text { QLQ-C30: } \\
\text { global health }\end{array}$ & $\begin{array}{l}\text { QLQ-C30: } \\
\text { functioning }\end{array}$ & $\begin{array}{l}\text { QLQ-C30: } \\
\text { symptoms }\end{array}$ \\
\hline \multicolumn{6}{|c|}{ Sex } \\
\hline Male $(\mathrm{N}=28)$ & $\begin{array}{c}65.8 \\
(30.1-97.0)\end{array}$ & $\begin{array}{c}71.9 \\
(46.7-88.4)\end{array}$ & $\begin{array}{c}70.7 \\
(33.3-100.0)\end{array}$ & $\begin{array}{c}89.5 \\
(55.3-100.0) \\
\end{array}$ & $\begin{array}{c}15.1 \\
(1.0-41.1)\end{array}$ \\
\hline Female $(\mathrm{N}=28)$ & $\begin{array}{c}64.4 \\
(14.5-94.2)\end{array}$ & $\begin{array}{c}72.6 \\
(51.6-86.9)\end{array}$ & $\begin{array}{c}71.1 \\
(7.5-100.0)\end{array}$ & $\begin{array}{c}87.9 \\
(57.4-100.0)\end{array}$ & $\begin{array}{c}15.5 \\
(0.0-52.0)\end{array}$ \\
\hline \multicolumn{6}{|c|}{ BMI } \\
\hline$<28(\mathrm{~N}=32)$ & $\begin{array}{c}67.6 \\
(29.4-96.4)\end{array}$ & $\begin{array}{c}72.9 \\
(47.5-87.2)\end{array}$ & $\begin{array}{c}71.4 \\
(10.8-100.0)\end{array}$ & $\begin{array}{c}89.0 \\
(50.9-100.0)\end{array}$ & $\begin{array}{c}13.5 \\
(0.8-36.8)\end{array}$ \\
\hline$\geq 28(\mathrm{~N}=24)$ & $\begin{array}{c}61.8 \\
(13.0-95.0)\end{array}$ & $\begin{array}{c}71.4 \\
(52.3-88.1)\end{array}$ & $\begin{array}{c}70.3 \\
(50.0-98.3)\end{array}$ & $\begin{array}{c}88.3 \\
(68.0-100.0)\end{array}$ & $\begin{array}{c}17.7 \\
(0.0-52.7)\end{array}$ \\
\hline \multicolumn{6}{|c|}{ Rheumatic disease and/or invalidity } \\
\hline No $(\mathrm{N}=23)$ & $\begin{array}{c}73.4 \text { * } \\
(21.6-97.5)\end{array}$ & $\begin{array}{c}72.8 \\
(49.1-88.1)\end{array}$ & $\begin{array}{c}78.3^{*} \\
(23.3-100.0)\end{array}$ & $\begin{array}{c}93.0 * \\
(73.9-100.0)\end{array}$ & $\begin{array}{c}8.9 * \\
(0.0-25.6)\end{array}$ \\
\hline Yes $(\mathrm{N}=33)$ & $\begin{array}{c}59.4 \text { * } \\
(19.1-93.9)\end{array}$ & $\begin{array}{c}71.9 \\
(48.1-87.8)\end{array}$ & $\begin{array}{c}65.6 * \\
(21.7-100.0)\end{array}$ & $\begin{array}{c}85.7^{*} \\
(50.9-100.0)\end{array}$ & $\begin{array}{c}19.7^{*} \\
(0.8-51.5)\end{array}$ \\
\hline \multicolumn{6}{|c|}{ Rheumatic disease } \\
\hline No $(\mathrm{N}=46)$ & $\begin{array}{c}68.1^{*} \\
(20.4-94.6)\end{array}$ & $\begin{array}{c}71.8 \\
(48.9-87.5)\end{array}$ & $\begin{array}{c}71.7 \\
(22.5-100.0)\end{array}$ & $\begin{array}{c}88.9 \\
(54.8-100.0)\end{array}$ & $\begin{array}{c}14.2 \\
(0.0-42.2)\end{array}$ \\
\hline Yes $(\mathrm{N}=10)$ & $\begin{array}{c}51.5^{*} \\
(23.0-76.5)\end{array}$ & $\begin{array}{c}74.6 \\
(51.9-85.8)\end{array}$ & $\begin{array}{c}66.7 \\
(33.3-83.3)\end{array}$ & $\begin{array}{c}87.7 \\
(67.3-96.0)\end{array}$ & $\begin{array}{c}20.4 \\
(1.9-25.0)\end{array}$ \\
\hline \multicolumn{6}{|c|}{ Invalidity } \\
\hline No $(\mathrm{N}=36)$ & $\begin{array}{c}68.0 \\
(16.9-95.9)\end{array}$ & $\begin{array}{c}72.8 \\
(50.9-88.4)\end{array}$ & $\begin{array}{c}72.5 \\
(14.2-100.0)\end{array}$ & $\begin{array}{c}90.7 \\
(70.2-100.0)\end{array}$ & $\begin{array}{c}13.1 \\
(0.0-51.0)\end{array}$ \\
\hline Yes $(N=20)$ & $\begin{array}{c}60.0 \\
(25.3-91.8)\end{array}$ & $\begin{array}{c}71.3 \\
(45.5-87.5)\end{array}$ & $\begin{array}{c}68.0 \\
(33.3-100.0)\end{array}$ & $\begin{array}{c}85.1 \\
(49.3-100.0)\end{array}$ & $\begin{array}{c}19.3 \\
(0.0-31.5)\end{array}$ \\
\hline
\end{tabular}

${ }^{1}$ Values of the components are expressed as arithmetic mean supplied with 5th-95th percentile range (in parentheses). SF-36 and QLQ -C30 were Scored according to the valid recommendations [11]

* Significantly different values between two categories given by the examined factor ( $p<0.05$; ANCOVA adjusted for age as continuous variable)

Table 2: Influence of examined cofactors on SF-36 and QLQ-C30 components'. 
Citation: Skrivanova K, Bendova M, Dusek L, Zackova D, Racil Z, et al. (2013) The Effect of Imatinib Treatment Duration on the Quality of the Life of Patients with Chronic Myeloid Leukemia. J Blood Disorders Transf 4: 167. doi: 10.4172/2155-9864.1000167

Page 4 of 5

\begin{tabular}{|c|c|c|c|c|c|c|}
\hline \multirow[b]{2}{*}{ Parameter } & \multicolumn{3}{|c|}{ Without invalidity and/or rheumatic disease $(\mathbf{n}=\mathbf{2 3})^{2}$} & \multicolumn{3}{|c|}{ With comorbidities $(n=33)^{2}$} \\
\hline & $\begin{array}{l}\text { Group A } \\
(n=11)\end{array}$ & $\begin{array}{c}\text { Group B } \\
(n=12)\end{array}$ & $p$ value $^{3}$ & $\begin{array}{c}\text { Group A } \\
(\mathrm{n}=17)\end{array}$ & $\begin{array}{c}\text { Group B } \\
(n=16)\end{array}$ & $p$ value $^{3}$ \\
\hline \multicolumn{7}{|c|}{ SF-36 transformed scores } \\
\hline Physical components-total & $\begin{array}{c}59.3 \\
(17.8-98.0)\end{array}$ & $\begin{array}{c}86.3 \\
(71.0-95.5)\end{array}$ & 0.001 & $\begin{array}{c}54.3 \\
(11.8-95.5)\end{array}$ & $\begin{array}{c}64.8 \\
(23.0-93.0)\end{array}$ & 0.218 \\
\hline Physical Function & 74.3 & $\begin{array}{c}91.7 \\
(80.0-100.0)\end{array}$ & 0.031 & $\begin{array}{c}53.4 \\
(0.0-95.0)\end{array}$ & $\begin{array}{c}70.9 \\
(20.0-95.0)\end{array}$ & 0.085 \\
\hline Role Physical & $\begin{array}{c}52.3 \\
(0.0-100.0)\end{array}$ & $\begin{array}{c}95.8 \\
(75.0-100.0)\end{array}$ & 0.004 & $\begin{array}{c}53.1 \\
(0.0-100.0)\end{array}$ & $\begin{array}{c}68.8 \\
(0.0-100.0)\end{array}$ & 0.22 \\
\hline Bodily Pain & $\begin{array}{c}66.6 \\
(31.0-100.0)\end{array}$ & $\begin{array}{c}87.5 \\
(62.0-100.0)\end{array}$ & 0.021 & $\begin{array}{c}67.8 \\
(22.0-100.0)\end{array}$ & $\begin{array}{c}69.3 \\
(0.0-100.0)\end{array}$ & 0.969 \\
\hline General Health & $\begin{array}{c}44 \\
(20.0-97.0)\end{array}$ & $\begin{array}{c}70.2 \\
(52.0-87.0)\end{array}$ & 0.001 & $\begin{array}{c}45.1 \\
(10.0-87.0)\end{array}$ & $\begin{array}{c}50 \\
(15.0-77.0)\end{array}$ & 0.456 \\
\hline Mental components - total & $\begin{array}{c}66.6 \\
(48.6-89.0)\end{array}$ & $\begin{array}{c}78.4 \\
(68.4-84.5)\end{array}$ & 0.015 & $\begin{array}{c}75.2 \\
(53.3-88.3)\end{array}$ & $\begin{array}{c}68.5 \\
(45.5-87.5)\end{array}$ & 0.132 \\
\hline Vitality & $\begin{array}{c}61.2 \\
30.0-100.0)\end{array}$ & $\begin{array}{c}75.4 \\
(60.0-90.0)\end{array}$ & 0.06 & $\begin{array}{c}62.5 \\
(30.0-90.0)\end{array}$ & $\begin{array}{c}63.7 \\
(5.0-95.0)\end{array}$ & 0.851 \\
\hline Social Function & $\begin{array}{c}67.7 \\
(60.0-95.0)\end{array}$ & $\begin{array}{c}64.6 \\
(60.0-80.0)\end{array}$ & 0.439 & $\begin{array}{c}72.4 \\
(60.0-90.0)\end{array}$ & $\begin{array}{c}68.8 \\
(60.0-95.0)\end{array}$ & 0.285 \\
\hline Role Emotional & $\begin{array}{c}70.4 \\
(0.0-100.0)\end{array}$ & $\begin{array}{c}91.7 \\
(33.0-100.0)\end{array}$ & 0.097 & $\begin{array}{c}86.3 \\
(33.0-100.0)\end{array}$ & $\begin{array}{c}66.7 \\
(0.0-100.0)\end{array}$ & 0.086 \\
\hline Mental Health & $\begin{array}{c}69.5 \\
(36.0-96.0)\end{array}$ & $\begin{array}{c}82 \\
(72.0-88.0)\end{array}$ & 0.047 & $\begin{array}{c}79.5 \\
(40.0-100.0)\end{array}$ & $\begin{array}{c}75.1 \\
(48.0-95.0)\end{array}$ & 0.498 \\
\hline \multicolumn{7}{|c|}{ QLQ-C30 transf. scores } \\
\hline Global health status in total & $\begin{array}{c}68.2 \\
(16.7-100.0)\end{array}$ & $\begin{array}{c}87.5 \\
(75.0-100.0)\end{array}$ & 0.012 & $\begin{array}{c}64.1 \\
(33.3-100.0)\end{array}$ & $\begin{array}{c}67.2 \\
(0.0-100.0)\end{array}$ & 0.218 \\
\hline Functioning in total & $\begin{array}{c}90.8 \\
(70.7-100.0)\end{array}$ & $\begin{array}{c}95 \\
(86.7-100.0)\end{array}$ & 0.13 & $\begin{array}{c}83 \\
(49.3-100.0)\end{array}$ & $\begin{array}{c}88.6 \\
(51.7-100.0)\end{array}$ & 0.085 \\
\hline Physical functioning & $\begin{array}{c}95.8 \\
(87.0-100.0)\end{array}$ & $\begin{array}{c}98.3 \\
(93.0-100.0)\end{array}$ & 0.106 & $\begin{array}{c}89 \\
(67.0-100.0)\end{array}$ & $\begin{array}{c}95 \\
(87.0-100.0)\end{array}$ & 0.22 \\
\hline Role functioning & $\begin{array}{c}86.4 \\
(67.0-100.0)\end{array}$ & $\begin{array}{c}97.2 \\
(83.0-100.0)\end{array}$ & 0.03 & $\begin{array}{c}75.5 \\
(33.0-100.0)\end{array}$ & $\begin{array}{c}87.5 \\
(33.0-100.0)\end{array}$ & 0.969 \\
\hline Emotional functioning & $\begin{array}{c}93.2 \\
(66.7-100.0)\end{array}$ & $\begin{array}{c}92.1 \\
(66.7-100.0)\end{array}$ & 0.795 & $\begin{array}{c}90.1 \\
(66.7-100.0)\end{array}$ & $\begin{array}{c}85.4 \\
(58.3-100.0)\end{array}$ & 0.456 \\
\hline Cognitive functioning & $\begin{array}{c}89.4 \\
(50.0-100.0)\end{array}$ & $\begin{array}{c}94.4 \\
(83.3-100.0)\end{array}$ & 0.344 & $\begin{array}{c}79.2 \\
(16.7-100.0)\end{array}$ & $\begin{array}{c}86.5 \\
(66.7-100.0)\end{array}$ & 0.132 \\
\hline Social functioning & $\begin{array}{c}89.4 \\
(67.0-100.0)\end{array}$ & $\begin{array}{c}93.1 \\
(67.0-100.0)\end{array}$ & 0.558 & $\begin{array}{c}80.2 \\
(17.0-100.0)\end{array}$ & $\begin{array}{c}88.5 \\
(0.0-100.0)\end{array}$ & 0.851 \\
\hline Symptoms. problems in total & $\begin{array}{c}12.2 \\
(3.7-25.9)\end{array}$ & $\begin{array}{c}6 \\
(0.0-16.7)\end{array}$ & 0.047 & $\begin{array}{c}20.7 \\
(1.2-53.2)\end{array}$ & $\begin{array}{c}18.7 \\
(0.0-50.6)\end{array}$ & 0.285 \\
\hline Fatigue & $\begin{array}{c}18.7 \\
(0.0-33.0)\end{array}$ & $\begin{array}{c}17.6 \\
(0.0-33.0)\end{array}$ & 0.859 & $\begin{array}{c}36.3 \\
(0.0-78.0)\end{array}$ & $\begin{array}{c}28.5 \\
(0.0-89.0)\end{array}$ & 0.086 \\
\hline Nausea and vomiting & $\begin{array}{c}7.6 \\
(0.0-33.3)\end{array}$ & 0 & 0.075 & $\begin{array}{c}15.7 \\
(0.0-83.3)\end{array}$ & $\begin{array}{c}9.4 \\
(0.0-16.7)\end{array}$ & 0.498 \\
\hline Pain & $\begin{array}{c}16.7 \\
(0.0-33.0)\end{array}$ & $\begin{array}{c}2.8 \\
(0.0-17.0)\end{array}$ & 0.009 & $\begin{array}{c}29.4 \\
(0.0-83.0)\end{array}$ & $\begin{array}{c}20.8 \\
(0.0-67.0)\end{array}$ & 0.819 \\
\hline Dyspnea & $\begin{array}{c}12.1 \\
(0.0-33.0)\end{array}$ & $\begin{array}{c}11.1 \\
(0.0-33.0)\end{array}$ & 0.879 & $\begin{array}{c}19.6 \\
(0.0-67.0)\end{array}$ & $\begin{array}{c}15.6 \\
(0.0-67.0)\end{array}$ & 0.203 \\
\hline Insomnia & $\begin{array}{c}27.3 \\
(0.0-66.7)\end{array}$ & $\begin{array}{c}8.3 \\
(0.0-33.3)\end{array}$ & 0.018 & $\begin{array}{c}27.5 \\
(0.0-100.0)\end{array}$ & $\begin{array}{c}37.8 \\
(0.0-100.0)\end{array}$ & 0.084 \\
\hline Appetite loss & 0 & 0 & NA & $\begin{array}{c}12.5 \\
(0.0-67.0)\end{array}$ & $\begin{array}{c}6.3 \\
(0.0-33.0)\end{array}$ & 0.077 \\
\hline Constipation & $\begin{array}{c}9.1 \\
(0.0-67.0)\end{array}$ & 0 & 0.164 & $\begin{array}{c}5.9 \\
(0.0-67.0)\end{array}$ & $\begin{array}{c}4.2 \\
(0.0-33.0)\end{array}$ & 0.528 \\
\hline Diarrhea & $\begin{array}{c}12.1 \\
(0.0-33.3)\end{array}$ & $\begin{array}{c}13.9 \\
(0.0-33.3)\end{array}$ & 0.8 & $\begin{array}{c}13.3 \\
(0.0-66.7)\end{array}$ & $\begin{array}{c}29.2 \\
(0.0-100.0)\end{array}$ & 0.378 \\
\hline Financial difficulties & $\begin{array}{c}6.1 \\
(0.0-33.0)\end{array}$ & 0 & 0.143 & $\begin{array}{c}18.8 \\
(0.0-100.0)\end{array}$ & $\begin{array}{c}16.7 \\
(0.0-100.0)\end{array}$ & 0.542 \\
\hline
\end{tabular}

${ }^{1}$ ANCOVA model with patients groups as factor and age as continuous covariate was adopted for the analysis

${ }^{2}$ Score values expressed as arithmetic mean with min-max values (in parentheses)

${ }^{3}$ Statistically significant results are given in bold $(p<0.05)$

Table 3: Comparison of SF-36/QLQ-C30 scores between group A-patients treated with Imatinib for 15 months (median) and group B-patients treated with Imatinib for 50 months (median) adjusted for age in the ANCOVA model'1. 
Citation: Skrivanova K, Bendova M, Dusek L, Zackova D, Racil Z, et al. (2013) The Effect of Imatinib Treatment Duration on the Quality of the Life of Patients with Chronic Myeloid Leukemia. J Blood Disorders Transf 4: 167. doi: 10.4172/2155-9864.1000167

in $\mathrm{p}$ values of $0.19-0.82$ (factors scored at the time of diagnosis), 0.11 0.81 (factors scored during Imatinib therapy), and 0.2-0.98 for factors scored at the time of testing.

The second aim of our study was to analyze the frequency and severity of depression in patients with CP-CML using the Beck Depression Inventory (BDI). We found that $61 \%$ of patients receiving short-term Imatinib treatment were depressed compared to $25 \%$ of the patients receiving Imatinib for a longer time. However, this difference was not statistically significant $(\mathrm{p}=0.406)$. Importantly, however, this difference was significant $(\mathrm{p}=0.046)$ in patients with invalidity and/or rheumatic disease. A significantly higher grade of depression severity was found in patients with these comorbidities and who had received Imatinib for a shorter duration of time, which confirmed our results found in the QL part of our study.

The main objective limitation of the study is the size of the examined group which limits the power of conclusions. To minimize the risk of confounding bias, we stratified the sample and the QL estimates according to several potentially confounding factors (sex, BMI, invalidity, etc.) and thus we tried to offer more detailed descriptive view into the QL profiles. All the outcomes are kept in the form of simple summary statistics, with directly presented data.

\section{Conclusion}

In summary, our study represents one of the first to analyze QL in patients with CML treated with Imatinib. We found a trend in the improvement in the QL of patients with CML in relation to the duration of Imatinib therapy. However, a statistically significant improvement of QL in patients with longer Imatinib treatment was only found in patients without co-existing invalidity and/or rheumatic disease. Moreover these two conditions increased the level of depressive symptoms in patients with CML during the early period of Imatinib therapy.

\section{Acknowledgement}

This study was supported by the Czech Leukemia Study Group for Life (CELL) and grant MSM0021622430. The authors thank Mgr. Libuse Kalvodova for her great help.

\section{References}

1. Ferrell BR, Dow KH, Grant M (1995) Measurement of the quality of life in cancer survivors. Qual Life Res 4: 523-531.

2. Scieszka M, Zielinski M, Machalski M, Herman ZS (2000) Quality of life in cancer patients treated by chemotherapy. Neoplasma 47: 396-399.

3. Houts AC, Lipinski D, Olsen JP, Baldwin S, Hasan M (2010) Use of the Patient Care Monitor to screen for depression in adult cancer patients interviewed with the structured clinical interview for DSM-IV. Psychooncology 19: 399-407.

4. Rowley JD (1973) Letter: A new consistent chromosomal abnormality in chronic myelogenous leukaemia identified by quinacrine fluorescence and Giemsa staining. Nature 243: 290-293.

5. Verfaillie CM (1998) Chronic myelogenous leukemia: too much or too little growth, or both? Leukemia 12: 136-138.

6. Druker BJ, Tamura S, Buchdunger E, Ohno S, Segal GM, et al. (1996) Effects of a selective inhibitor of the Abl tyrosine kinase on the growth of Bcr-Abl positive cells. Nat Med 2: 561-566.

7. le Coutre P, Mologni L, Cleris L, Marchesi E, Buchdunger E, et al. (1999) In

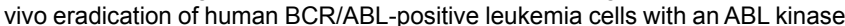
inhibitor. J Natl Cancer Inst 91: 163-168.
8. Druker BJ, Guilhot F, O'Brien SG, Gathmann I, Kantarjian H, et al. (2006) Fiveyear follow-up of patients receiving imatinib for chronic myeloid leukemia. $\mathrm{N}$ Engl J Med 355: 2408-2417.

9. Hochhaus A, O'Brien SG, Guilhot F, Druker BJ, Branford S, et al. (2009) Sixyear follow-up of patients receiving imatinib for the first-line treatment of chronic myeloid leukemia. Leukemia 23: 1054-1061.

10. Baccarani M, Saglio G, Goldman J, Hochhaus A, Simonsson B, et al (2006) Evolving concepts in the management of chronic myeloid leukemia: recommendations from an expert panel on behalf of the European LeukemiaNet. Blood 108: 1809-1820

11. Baccarani M, Cortes J, Pane F, Niederwieser D, Saglio G, et al. (2009) Chronic myeloid leukemia: an update of concepts and management recommendations of European LeukemiaNet. J Clin Oncol 27: 6041-6051.

12. Hahn EA, Glendenning GA, Sorensen MV, Hudgens SA, Druker BJ, et al (2003) Quality of life in patients with newly diagnosed chronic phase chronic myeloid leukemia on imatinib versus interferon alfa plus low-dose cytarabine: results from the IRIS Study. J Clin Oncol 21: 2138-2146.

13. Osoba D, Aaronson N, Zee B, Sprangers M, te Velde A (1997) Modification of the EORTC QLQ-C30 (version 2.0) based on content validity and reliability testing in large samples of patients with cancer. The Study Group on Quality of Life of the EORTC and the Symptom Control and Quality of Life Committees of the $\mathrm{NCl}$ of Canada Clinical Trials Group. Qual Life Res 6: 103-108.

14. Aaronson NK, Ahmedzai S, Bergman B, Bullinger M, Cull A, et al. (1993) The European Organization for Research and Treatment of Cancer QLQ-C30: a quality-of-life instrument for use in international clinical trials in oncology. J Natl Cancer Inst 85: 365-376.

15. Ware JE, Sherbourne CD (1992) The MOS 36-item short form health survey (SF-36). I. Conceptual framework and item selection. Med Care 30: 473-483.

16. Osoba D, Rodrigues G, Myles J, Zee B, Pater J (1998) Interpreting the significance of changes in health-related quality-of-life scores. J Clin Oncol 16 : 139-144.

17. Beck AT, Ward CH, Mendelsohn M, Mock J, Erbaugh J (1961) An inventory for measuring depression. Arch Gen Psychiatry 4: 561-571.

18. SPSS for Windows (2003) SPSS Inc, Chicago.

19. Bayliss EA, Ellis JL, Steiner JF (2005) Subjective assessments of comorbidity correlate with quality of life health outcomes: initial validation of a comorbidity assessment instrument. Health Qual Life Outcomes 3: 51.

Citation: Skrivanova K, Bendova M, Dusek L, Zackova D, Racil Z, et al. (2013) The Effect of Imatinib Treatment Duration on the Quality of the Life of Patients with Chronic Myeloid Leukemia. J Blood Disorders Transf 4: 167. doi: 10.4172/2155-9864.1000167 